\title{
Neutron reactions and nuclear cosmo-chronology
}

M. Mosconi ${ }^{1}$, M. Heil ${ }^{1,2}$, F. Käppeler ${ }^{1, *}$, A. Mengoni ${ }^{3}$, R. Plag ${ }^{1}$, K. Fujii ${ }^{4}$, R. Gallino ${ }^{5}$, G. Aerts ${ }^{6}$, R. Terlizzi ${ }^{7}$, U. Abbondanno ${ }^{4}$, H. Álvarez-Pol ${ }^{12}$, F. Alvarez-Velarde ${ }^{6}$, S. Andriamonje ${ }^{6}$, J. Andrzejewski ${ }^{19}$, P. Assimakopoulos ${ }^{8}$, L. Audouin ${ }^{6}$, G. Badurek ${ }^{9}$, P. Baumann ${ }^{10}$, F. Bečváŕr ${ }^{11}$, E. Berthoumieux ${ }^{6}$, S. Bisterzo $^{5,1}$, F. Calviño ${ }^{13}$, D. Cano-Ott ${ }^{14}$, R. Capote ${ }^{3,15}$, A. Carrillo de Albornoz ${ }^{16}$, P. Cennini ${ }^{17}$, V. Chepel ${ }^{18}$, E. Chiaveri ${ }^{17}$, N. Colonna ${ }^{7}$, G. Cortes ${ }^{13}$, A. Couture ${ }^{20}$, J. Cox ${ }^{20}$, M. Dahlfors ${ }^{17}$, S. David ${ }^{21}$, I. Dillman ${ }^{1}$, R. Dolfini ${ }^{22}$, C. Domingo Pardo ${ }^{1}$, W. Dridi ${ }^{14}$, I. Duran $^{12}$, C. Eleftheriadis ${ }^{23}$, M. Embid-Segura ${ }^{14}$, L. Ferrant ${ }^{21}$, A. Ferrari ${ }^{17}$, R. Ferreira-Marques ${ }^{18}$, L. Fitzpatrick ${ }^{17}$, H. Frais-Koelbl ${ }^{6}$, W. Furman ${ }^{24}$, I. Goncalves ${ }^{18}$, E. Gonzalez-Romero ${ }^{14}$, A. Goverdovski ${ }^{25}$, F. Gramegna ${ }^{26}$, E. Griesmayer ${ }^{6}$, C. Guerrero ${ }^{14}$, F. Gunsing ${ }^{6}$, B. Haas ${ }^{27}$,

R. Haight ${ }^{28}$, A. Herrera-Martinez ${ }^{17}$, M. Igashira ${ }^{29}, \mathrm{~S}$. Isaev ${ }^{21}$, E. Jericha ${ }^{9}$, Y. $\operatorname{Kadi}^{17}$, D. Karamanis ${ }^{8}$, D. Karadimos ${ }^{8}$, M. Kerveno ${ }^{10}$, V. Ketlerov ${ }^{25,17}$, P. Koehler ${ }^{30}$, V. Konovalov ${ }^{24,17}$, E. Kossionides ${ }^{31}$, M. Krtička ${ }^{11}$, C. Lamboudis ${ }^{8}$, H. Leeb ${ }^{9}$, A. Lindote ${ }^{18}$, I. Lopes ${ }^{18}$, M. Lozano ${ }^{15}$, S. Lukic ${ }^{10}$, J. Marganiec ${ }^{19}$, L. Marques ${ }^{16}$, S. Marrone ${ }^{7}$, P. Mastinu ${ }^{26}$, P.M. Milazzo ${ }^{4}$, C. Moreau ${ }^{4}$, F. Neves ${ }^{18}$, H. Oberhummer ${ }^{9}$, M. Oshima ${ }^{32}$, S. O’Brien ${ }^{20}$, J. Pancin ${ }^{6}$, C. Papachristodoulou ${ }^{8}$, C. Papadopoulos ${ }^{33}$, C. Paradela ${ }^{12}$, N. Patronis ${ }^{8}$, A. Pavlik ${ }^{34}$, P. Pavlopoulos ${ }^{35}$, L. Perrot ${ }^{6}$, A. Plompen ${ }^{36}$, A. Plukis ${ }^{6}$, A. Poch ${ }^{13}$, C. Pretel ${ }^{13}$, J. Quesada ${ }^{15}$, T. Rauscher ${ }^{37}$, R. Reifarth ${ }^{28}$, M. Rosetti ${ }^{38}$, C. Rubbia ${ }^{22}$, G. Rudolf ${ }^{10}$, P. Rullhusen ${ }^{36}$, J. Salgado ${ }^{16}$, L. Sarchiapone ${ }^{17}$, I. Savvidis ${ }^{23}$, C. Stephan ${ }^{21}$, G. Tagliente ${ }^{7}$, J.L. Tain ${ }^{40}$, 
L. Tassan-Got ${ }^{21}$, L. Tavora ${ }^{16}$, G. Vannini ${ }^{39}$, P. Vaz ${ }^{16}$, A. Ventura ${ }^{38}$, D. Villamarin ${ }^{6}$, M.C. Vincente ${ }^{6}$, V. Vlachoudis ${ }^{17}$, R. Vlastou ${ }^{33}$, F. Voss ${ }^{1}$, S. Walter ${ }^{1}$, H. Wendler ${ }^{17}$, M. Wiescher ${ }^{20}$, K. Wisshak ${ }^{1}$

${ }^{1}$ Forschungszentrum Karlsruhe GmbH (FZK), Institut für Kernphysik, Germany

${ }^{2}$ Gesellschaft für Schwerionenforschung (GSI), Germany

${ }^{3}$ International Atomic Energy Agency, NAPC-Nuclear Data Section, Vienna, Austria

${ }^{4}$ Istituto Nazionale di Fisica Nucleare, Trieste, Italy

${ }^{5}$ Dipartimento di Fisica Generale, Università di Torino, Italy

${ }^{6}$ CEA/Saclay - DSM, Gif-sur-Yvette, France

${ }^{7}$ Istituto Nazionale di Fisica Nucleare, Bari, Italy

${ }^{8}$ University of Ioannina, 45110 Ioannina, Greece

${ }^{9}$ Atominstitut der Österreichischen Universitäten, Technische Universität Wien, Austria

${ }^{10}$ Centre National de la Recherche Scientifique/IN2P3 - IReS, Strasbourg, France

${ }^{11}$ Charles University, CZ-180 00 Prague, Czech Republic

12 Universidad de Santiago de Compostela, 15706 Santiago de Compostela, Spain

13 Universitat Politecnica de Catalunya, 08028 Barcelona, Spain

${ }^{14}$ Centro de Investigaciones Energeticas Medioambientales y Technologicas, Madrid, Spain

${ }^{15}$ Universidad de Sevilla, Spain

${ }^{16}$ Instituto Tecnológico e Nuclear(ITN), Lisbon, Portugal

${ }^{17}$ CERN, 1211 Geneva 23, Switzerland

${ }^{18}$ LIP - Coimbra \& Departamento de Fisica da Universidade de Coimbra, Portugal

${ }^{19}$ University of Lodz, 90-236 Lodz, Poland

${ }^{20}$ University of Notre Dame, Notre Dame, USA

${ }^{21}$ Centre National de la Recherche Scientifique/IN2P3 - IPN, Orsay, France

${ }^{22}$ Università degli Studi Pavia, Pavia, Italy

${ }^{23}$ Aristotle University of Thessaloniki, 54006 Thessaloniki, Greece

24 Joint Institute for Nuclear Research, Frank Laboratory of Neutron Physics, Dubna, Russia

${ }^{25}$ Institute of Physics and Power Engineering, Kaluga region, Obninsk, Russia

${ }^{26}$ Istituto Nazionale di Fisica Nucleare(INFN), Laboratori Nazionali di Legnaro, Italy

${ }^{27}$ Centre National de la Recherche Scientifique/IN2P3 - CENBG, Bordeaux, France

${ }^{28}$ Los Alamos National Laboratory, Los Alamos, NM, USA

${ }^{29}$ Tokyo Institute of Technology, Tokyo, Japan

${ }^{30}$ Oak Ridge National Laboratory, Physics Division, Oak Ridge, TN, USA

${ }^{31}$ National Center for Scientific Research, "Demokritos NCSR”, Athens, Greece

32 Japan Atomic Energy Research Institute, Tokai-mura, Japan

33 National Technical University of Athens, Greece

${ }^{34}$ Institut für Isotopenforschung und Kernphysik, Universität Wien, Austria

${ }^{35}$ Pôle Universitaire Léonard de Vinci, Paris La Défense, France

${ }^{36}$ CEC-JRC-IRMM, Geel, Belgium

${ }^{37}$ Department of Physics and Astronomy, University of Basel, Basel, Switzerland

${ }^{38}$ ENEA, 40128 Bologna, Italy

${ }^{39}$ Dipartimento di Fisica, Università di Bologna, and Sezione INFN di Bologna, Italy

${ }^{40}$ Instituto de Fisica Corpuscular, CSIC-Universidad de Valencia, Valencia, Spain

\footnotetext{
* Corresponding author.

E-mail address: franz.kaeppeler@ik.fzk.de (F. Käppeler).
} 


\begin{abstract}
The $\beta$-decay of ${ }^{187} \operatorname{Re}\left(t_{1 / 2}=42.3 \mathrm{Gyr}\right)$ represents a suited cosmo-chronometer for the age of the $r$-process abundances, since the radiogenic part of the daughter isotope ${ }^{187} \mathrm{Os}$ is defined by the difference between the solar ${ }^{187}$ Os abundance and $s$-process contribution to ${ }^{187} \mathrm{Os}$. The latter component can be determined via the $s$-process systematics based on the stellar neutron capture cross sections of ${ }^{186} \mathrm{Os}$ and ${ }^{187}$ Os. The laboratory cross section of ${ }^{187}$ Os requires a significant correction for the effect of the low-lying excited state at $9.75 \mathrm{keV}$, which is strongly populated under stellar conditions. This theoretical correction can be improved by an experimental cross section for inelastic scattering to the $9.75 \mathrm{keV}$ state. High resolution time-of-flight measurements of $(n, \gamma)$ cross sections of ${ }^{186,187,188} \mathrm{Os}$ from $1 \mathrm{eV}$ to $1 \mathrm{MeV}$ at CERN $n_{-}$TOF facility are reported. The inferred stellar cross sections differ from previously recommended values. In addition, the inelastic scattering cross section has been measured at $30 \mathrm{keV}$ neutron energy via time-of-flight at the Karlsruhe 3.7 MV Van de Graaff. The implications of these results for the Re/Os clock are discussed.
\end{abstract}

(c) 2007 Elsevier B.V. All rights reserved.

Keywords: Re/Os cosmo-chronometer; $s$ process; Stellar cross sections; Neutron capture; Inelastic scattering

\title{
1. Introduction
}

The long half-life of 42.3 Gyr makes ${ }^{187} \mathrm{Re}$ an important potential cosmo-chronometer. Originally proposed by Clayton [1], this chronometer is particularly appealing because ${ }^{187} \operatorname{Re}$ can be attributed to the $r$ process, which is believed to occur in supernova explosions of massive stars. Since these stars evolve quickly, the clock started early after galaxy formation. Another important feature of this clock is that it can be almost completely analyzed in terms of the nuclear properties of the mother/daughter pair ${ }^{187} \mathrm{Re} /{ }^{187} \mathrm{Os}$. Therefore, it represents a valuable complement to other dating methods based on astronomical observations, i.e. for deriving the Hubble age [2], globular cluster ages [3], or age estimates from analyses of the cosmic microwave background [4].

Problems related to the Re/Os clock (apart from a realistic model of galactic chemical evolution) were found to originate from the dramatic temperature dependence of the ${ }^{187} \mathrm{Re}$ half-life, a possible minor $s$-process contribution to ${ }^{187} \mathrm{Re}$, and a sizable correction for the $s$ process abundance component of ${ }^{187} \mathrm{Os}$ due to thermal population of excited nuclear states in this odd-even isotope. The first issue could be settled by a measurement of the half-life of fully stripped ${ }^{187} \mathrm{Re}$ atoms [5] and by subsequent analyses, which demonstrated that the astration effect related to the destruction of ${ }^{187} \mathrm{Re}$ in later stellar generations is not crucial for the reliability of the clock [6]. Similarly, the $s$-process contributions to ${ }^{187} \mathrm{Re}$ due to the branchings at ${ }^{185} \mathrm{~W}$ and ${ }^{186} \mathrm{Re}$ were shown to have no significant impact either [7-9].

The remaining nuclear issues, which need to be addressed for the improvement of the Re/Os clock, refer to the determination of more accurate Maxwellian averaged cross sections (MACS) for neutron capture on ${ }^{186} \mathrm{Os}$ and ${ }^{187} \mathrm{Os}$. This information is required for defining the $s$-process contribution to ${ }^{187}$ Os via the so-called local approximation, $\sigma N_{s}=$ const, which is well satisfied in this mass region.

Since ${ }^{186} \mathrm{Os}$ is exclusively produced by the $s$ process, the $s$ component of ${ }^{187} \mathrm{Os}$ is

$$
N_{s}^{187}{ }^{18 \mathrm{Os}}=\frac{\left\langle\sigma_{186} \mathrm{Os}\right\rangle}{\left\langle\sigma_{187}{ }_{\mathrm{Os}}\right\rangle} N_{s}^{186}{ }^{\mathrm{Os}},
$$



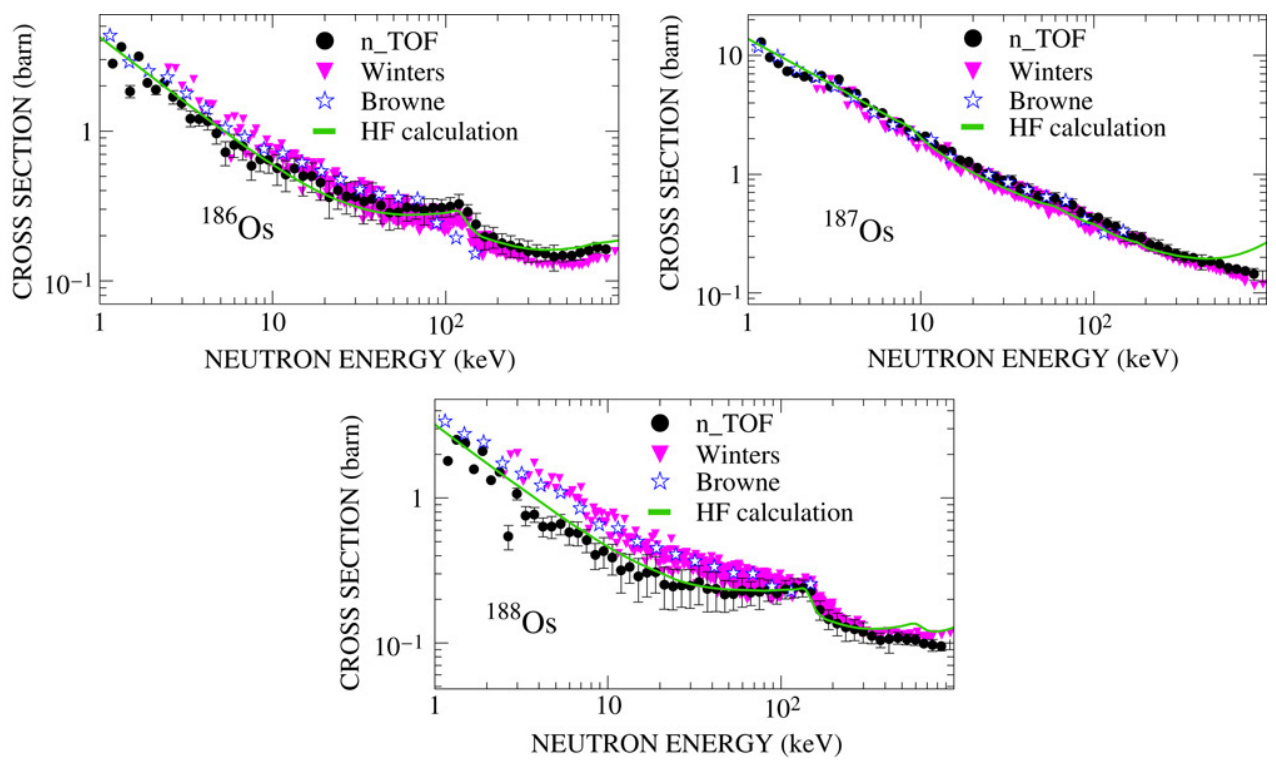

Fig. 1. Comparison between the present results (full circles with error bars) and the data of Winters et al. [11] (triangles) and of Browne and Berman [10] (stars), complemented by a recent statistical model calculation (solid line). The present results are composed of averaged cross sections obtained from detailed resonance analyses (below $3 \mathrm{keV}$ for ${ }^{186} \mathrm{Os}$ and ${ }^{188} \mathrm{Os}$ and below $2 \mathrm{keV}$ for ${ }^{187} \mathrm{Os}$ ) and from the continuum part at higher energies.

where $\left\langle\sigma_{i}\right\rangle$ are the MACSs that can in principle be calculated from experimentally measured $(n, \gamma)$ cross sections. Under stellar conditions, however, the MACS derived from laboratory data must be corrected for the effect of thermally populated excited states. These stellar enhancement factors have to be obtained by theory. In this case, additional knowledge of the inelastic cross section can be used to constrain such calculations. The stellar enhancement effect is particularly important for ${ }^{187} \mathrm{Os}$, which exhibits low-lying nuclear states at 9.8 and $74.3 \mathrm{keV}$.

Previous measurements of the neutron capture cross sections from ${ }^{186} \mathrm{Os}$ to ${ }^{190} \mathrm{Os}$ and of ${ }^{192}$ Os $[10,11]$ suffered from the fact that the astrophysically relevant energy range was not fully covered. This holds in particular for the low energy region, which is important for most of the $s$-process neutron exposure provided by the ${ }^{13} \mathrm{C}(\alpha, n){ }^{16} \mathrm{O}$ reaction [12]. Among the two cross section sets, the MACS of ${ }^{186} \mathrm{Os}$ exhibit some discrepancies, especially at energies close to the first excited state of ${ }^{186} \mathrm{Os}$ at $130 \mathrm{keV}$, where a Hauser-Feshbach calculation is suggesting a pronounced step in the cross section (Fig. 1). Consequently, improved capture cross section measurements are called for to resolve existing discrepancies and to extend the energy range to lower and higher energies for obtaining a more reliable energy dependence of the $(n, \gamma)$ cross sections.

Finally, the available measurements of the inelastic scattering cross section of ${ }^{187} \mathrm{Os}$ [13-15] also exhibit large uncertainties that are limiting the reliability of the calculated stellar enhancement factor and, hence, the Re/Os age. Therefore, an accurate independent measurement of the $\left(n, n^{\prime}\right)$ cross section of ${ }^{187}$ Os is called for as well.

In this contribution we report on new measurements of the neutron capture and inelastic scattering cross sections for an improved assessment of the Re/Os cosmo-chronometer. 


\section{Neutron capture cross sections}

The $(n, \gamma)$ cross sections of ${ }^{186,187,188}$ Os have been measured at the CERN/n_TOF spallation facility [16]. Neutrons are produced by means of a fast pulsed proton beam $(20 \mathrm{GeV}$ protons with $6 \mathrm{~ns}$ pulse width) impinging on a solid lead target, which provides a wide spectrum from thermal energies to above $1 \mathrm{GeV}$. Neutron capture measurements are commonly performed in a restricted energy range from $1 \mathrm{eV}$ to $1 \mathrm{MeV}$. Due to the long flight path of $187.5 \mathrm{~m}$ and the short proton pulse, the time of flight (TOF) method yields an excellent neutron energy resolution of $3 \times 10^{-4}$ and $4 \times 10^{-3}$ at $1 \mathrm{eV}$ and $1 \mathrm{MeV}$, respectively. The neutron flux varies smoothly with energy, reaching values of $4.5 \times 10^{4}-2 \times 10^{5}$ neutrons per energy decade per proton bunch between $1 \mathrm{eV}$ and $1 \mathrm{MeV}$ in the experimental area at $187.5 \mathrm{~m}$.

Each of the Os samples consisted of $2 \mathrm{~g}$ of enriched metal powder provided by Oak Ridge National Laboratory. In fact, this was the same material as used in previous experiments $[11,10]$. The ${ }^{186}$ Os and ${ }^{187}$ Os samples were enriched to $79 \%$ and $70 \%$ with ${ }^{188}$ Os contaminations of $5 \%$ and $\sim 13 \%$, respectively. Therefore, the $(n, \gamma)$ cross section of ${ }^{188}$ Os was measured as well using a $95 \%$ enriched sample in order to account for the main isotopic correction. The impurities due to ${ }^{189} \mathrm{Os},{ }^{190} \mathrm{Os}$, and ${ }^{192}$ Os were all around $5 \%$. The sample material was encapsulated in thin aluminum cans of diameter $15 \mathrm{~mm}$ in diameter.

The $\gamma$-ray cascades from capture events have been detected with two $\mathrm{C}_{6} \mathrm{D}_{6}$ scintillators $(12 \mathrm{~cm}$ in diameter and each with a volume of $1000 \mathrm{~cm}^{3}$ ), which were placed perpendicular to the neutron beam. A neutron monitor consisting of a thin ${ }^{6} \mathrm{Li}$ foil was used for flux normalization, which was surrounded by a set of four Si detectors mounted outside the neutron beam for recording the products of the ${ }^{6} \mathrm{Li}(n, \alpha)^{3} \mathrm{H}$ reaction. The $\gamma$-detectors were designed to minimize the neutron sensitivity [17]. Signals from the $\mathrm{C}_{6} \mathrm{D}_{6}$ scintillators were recorded with flash amplitude-to-digital converters (FADC) for off-line application of the pulse height weighting technique (PHWT) [18]. In this way, the detection efficiency can be corrected off-line to become independent of the multiplicity of the prompt capture $\gamma$-ray cascade. Since the PHWT requires a separate evaluation of the background, a set of additional samples was mounted on the ladder of a sample changer, i.e. a carbon sample for the effect of scattered neutrons, a lead sample for the scattering of in-beam $\gamma$-rays, a gold sample for neutron flux normalization, and an empty position for obtaining the ambient background.

All samples have been measured under the same beam conditions. The neutron flux normalization was achieved by means of the Si neutron monitor and by calibration runs with the gold sample. The cross sections were normalized to the gold cross section of Ref. [19] using the experimental average cross section for the quasi-stellar spectrum. The background components in the spectra of the Os samples and of the gold sample have been treated individually. In particular, the weighting functions were determined by a complete simulation of the experimental setup, including the effect of the sample itself, and the evaluated background has been validated by comparison with spectra obtained with black neutron filters in the beam.

The analysis of the data and the calculation of the cross section was separated in the resolved resonance region and in the continuum part. The resolved resonance region has been analyzed by the R-matrix code SAMMY. For ${ }^{186} \mathrm{Os},{ }^{187} \mathrm{Os}$, and ${ }^{188} \mathrm{Os}$ individual resonance parameters were obtained up to 3,2, and $5 \mathrm{keV}$ neutron energy, respectively. The background obtained by the SAMMY analysis was found to agree perfectly with the result of the sample changer technique. This confirms the background subtraction in an independent way.

In the continuum, the cross sections have been determined by subtracting the background and by normalizing the resulting data to the evaluated flux. Corrections were applied for self- 

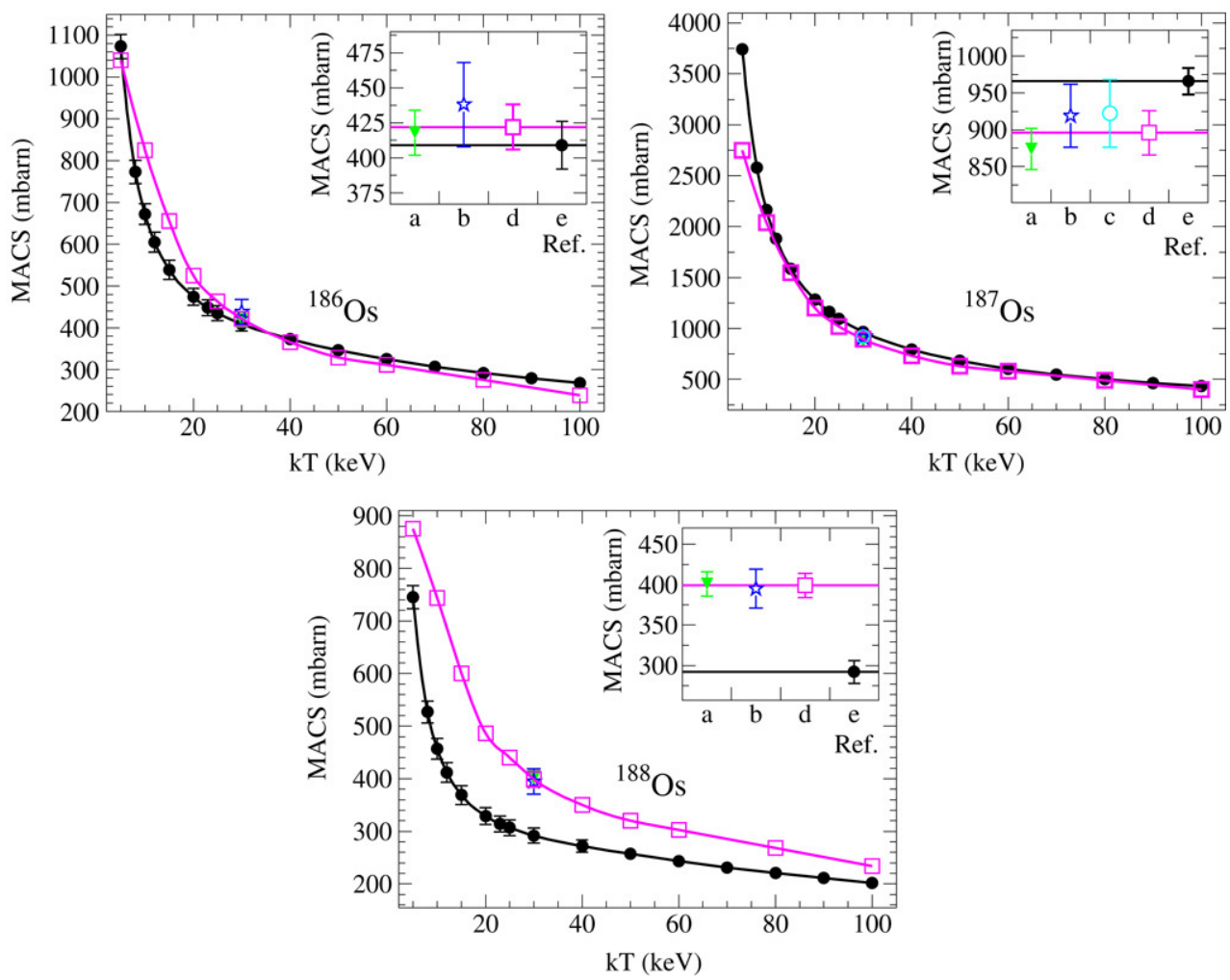

Fig. 2. Present Maxwellian averaged cross sections (solid circles) compared to the recommended data of Bao it et al. [21] (squares) and to previous experimental results of Winters et al. [11] (triangles), Browne and Berman [10] (stars), and Bokhovko et al. [22] (open circles). The insets focus on the values at $k T=30 \mathrm{keV}$, which are commonly used for comparison.

absorption and multiple scattering (calculated with the SESH code [20]), and for the respective impurities. The final cross sections plotted in Fig. 1 were calculated by combining the average data from the resonance analysis and from the continuum region. The comparison in Fig. 1 shows good agreement with previous data only for ${ }^{187} \mathrm{Os}$, whereas significant differences with respect to previous measurements are found for the even isotopes ${ }^{186} \mathrm{Os}$ and ${ }^{188} \mathrm{Os}$. Up to $400 \mathrm{keV}$ the present results are well described by the Hauser-Feshbach statistical model calculations.

The MACSs are evaluated for thermal energies from 5 to $100 \mathrm{keV}$ (Fig. 2). At $k T=30 \mathrm{keV}$, the present results for ${ }^{186}$ Os and ${ }^{188}$ Os are $9 \%$ and $30 \%$ lower than the respective recommended values [21], whereas that for ${ }^{187}$ Os is in agreement with the data of Browne and Berman [10] and of Bokhovko et al. [22]; compared to Ref. [21] it is 5\% higher, but still within the quoted uncertainties.

During the $s$ process reaction flow equilibrium has been locally established from the mass region of the rare earth elements up to $A=190$ due to the comparably large MACSs of the involved isotopes. This implies that the local approximation $N_{s} \times\langle\sigma\rangle=$ constant holds. Comparison of the $N_{s} \times\langle\sigma\rangle$ values of the two $s$-only isotopes ${ }^{186}$ Os and ${ }^{187}$ Os can, therefore, be used for deriving the excess ${ }^{187}$ Os from the measured ratio of the stellar MACS of ${ }^{187}$ Os and 
${ }^{186}$ Os. This excess corresponds to the radiogenic component due to the decay of the long-lived ${ }^{187} \mathrm{Re}$.

Based on the measured cross sections, the MACS ratio is $186 / 187=0.41 \pm 0.02$. Under the assumption of the coarse Fowler model [23] for galactic chemical evolution (GCE) and neglecting the time prior to the formation of our Galaxy, the age of the Universe is estimated to be around 15 Gyr. By including the stellar enhancement factor for the MACS of ${ }^{187}$ Os from Ref. [21], this ratio reduces to $0.35 \pm 0.02$, yielding $s$ slightly higher age of $16.5 \pm 2 \mathrm{Gyr}$, somewhat higher than the U/Th age of $14.5 \pm{ }_{2.2}^{2.8} \mathrm{Gyr}$ [24] and than the results based on cosmological evidence [4]. This very preliminary estimate has yet to be verified by means of an improved evaluation of the stellar enhancement factor and by using a more realistic galactic evolution model.

\section{Inelastic scattering on ${ }^{187} \mathrm{Os}$}

The neutron inelastic scattering cross section of ${ }^{187}$ Os was measured at the $3.7 \mathrm{MV}$ Van de Graaff of Forschungszentrum Karlsruhe using the ${ }^{7} \mathrm{Li}(p, n)^{7} \mathrm{Be}$ reaction for obtaining a 'monoenergetic' neutron beam of $30 \mathrm{keV}$. The energy of the pulsed proton beam $(\Delta t=10 \mathrm{~ns})$ was adjusted right at the $(p, n)$ threshold to produce kinematically collimated neutrons, which are emitted in a narrow cone with an opening angle of no more than $13 \mathrm{deg}$ and an energy spread of less than $10 \mathrm{keV}$. In this way, neutron collimators could be avoided, because the detectors for scattered neutrons were placed outside the main neutron beam. The $\gamma$-ray flash from the impact of the proton pulse on the ${ }^{7} \mathrm{Li}$ target was reduced by a small lead shield around the target.

The measurement had to be performed with the same ${ }^{187}$ Os and ${ }^{188}$ Os samples as used in the neutron capture experiment in order to avoid losses of the expensive material. The samples were mounted $4 \mathrm{~cm}$ in front of the target under an angle of $45 \mathrm{deg}$ in order to minimize self shielding effects. Scattered neutrons were detected by three ${ }^{6} \mathrm{Li}$-glass scintillators $3 \mathrm{~mm}$ in thickness and $11 \mathrm{~cm}$ in diameter, two at $90 \mathrm{deg}$ and one at $75 \mathrm{deg}$ with respect to the direction of the primary beam. All detectors were mounted $26 \mathrm{~cm}$ from the scattering sample. In addition, a $3 \mathrm{~mm}$ thick ${ }^{6} \mathrm{Li}$-glass scintillator $3.8 \mathrm{~cm}$ in diameter in the forward direction at $111 \mathrm{~cm}$ from the ${ }^{7} \mathrm{Li}$ target served as a monitor for the primary neutron flux. The data acquisition system consisted of a set of four FADCs, which had the advantage that the recorded signals could be analyzed in detail offline. In this way, the signal/background ratio could be improved by pulse shape discrimination between neutrons and $\gamma$-rays.

During the measurements the proton energy was tuned such that the FWHM of the neutron energy distribution was below $10 \mathrm{keV}$. Due to the normal charge fluctuations of the Van de Graaff, the width of the distribution varied between 7 and $10 \mathrm{keV}$ in cycles of several hours.

The separation of the elastic and inelastic scattering component was obtained by measuring the neutron energies via TOF and by using the elastic component from the scattering spectrum of ${ }^{188}$ Os. The inelastic part can then be extracted by GEANT simulations assuming that the inelastic component is represented by the same distribution shifted in energy by the $9.75 \mathrm{keV}$ excitation energy of the first excited state of ${ }^{187}$ Os. In this way, the fit contains only two free parameters, namely the amplitudes of the elastic and inelastic distributions. This analysis is not yet completed; a preliminary result is shown in Fig. 3.

In parallel to this work, cross section studies of neutron capture on ${ }^{186,187,188}$ Os and inelastic scattering on ${ }^{187}$ Os have been carried out with a different setup and over a restricted energy range [25], but results are not yet available. 


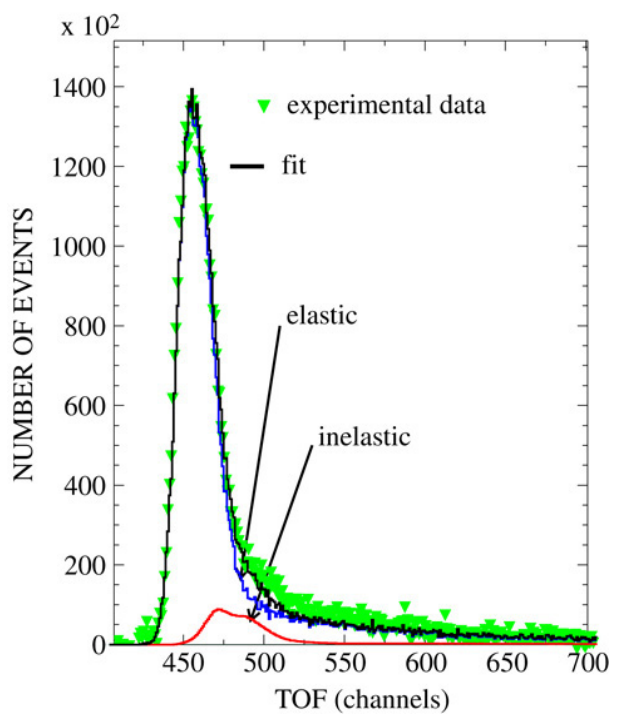

Fig. 3. Spectra of scattered neutrons from ${ }^{187}$ Os corresponding to a neutron beam of $9.5 \mathrm{keV}$ FWHM. The GEANT simulations (solid lines) of the measured data (triangles) have been obtained considering the full geometry of the experimental setup, which is reflected by the dip in the inelastic spectrum. The signature of the inelastic component in this still preliminary stage of data analysis suggests that the inelastic scattering cross section is more than 10 times smaller than the elastic part. The final value can only be given after the background above channel 500 is properly understood.

\section{Summary}

The challenges related to the neutron capture cross section measurements have been met: the $\mathrm{n}_{-} \mathrm{TOF}$ cross sections are available in the entire range of astrophysical interest with typical uncertainties of 5\%. The data exhibit clear signatures for the competition of the inelastic channel, which had previously not been found for ${ }^{186} \mathrm{Os}$. A final statement concerning the Re/Os clock must await completion of the analysis of the inelastic scattering cross section. At present, the data seem to indicate an older age of the universe than that obtained with other methods, but this may well be due to the very crude GCE model used so far.

\section{Acknowledgments}

This work is supported by National Institutions of the related n_TOF participants and partially by EC under contract FIKW-CT-2000-00107.

\section{References}

[1] D.D. Clayton, Astrophys. J. 139 (1964) 637.

[2] W.L. Freedman, et al., Astrophys. J. 553 (2001) 47.

[3] L.M. Krauss, B. Chaboyer, Science 299 (2003) 65.

[4] C.L. Bennett, et al., Astrophys. J. 148 (Suppl.) (2003) 1.

[5] F. Bosch, et al., Phys. Rev. Lett. 77 (1996) 5190.

[6] K. Takahashi, in: M. Arnould, M. Lewitowicz, Y. Oganessian, M. Ohta, H. Utsunomiya, T. Wada (Eds.), Tours Symposium on Nuclear Physics III, AIP, New York, 1998, p. 616.

[7] F. Käppeler, S. Jaag, Z.Y. Bao, G. Reffo, Astrophys. J. 366 (1991) 605. 
[8] T. Shizuma, et al., Phys. Rev. C 72 (2005) 025808.

[9] P. Mohr, S. Goriely, T. Shizuma, T. Hayakawa, in: A. Mengoni, et al. (Eds.), Nuclei in the Cosmos-IX (to be published in Proceedings of Science, http://pos.sissa.it).

[10] J.C. Browne, B.L. Berman, Phys. Rev. C 23 (1981) 1434.

[11] R.R. Winters, R.L. Macklin, J. Halperin, Astrophys. J. 233 (1979) 411.

[12] R. Gallino, C. Arlandini, M. Busso, M. Lugaro, C. Travaglio, O. Straniero, A. Chieffi, M. Limongi, Astrophys. J. 497 (1998) 388.

[13] R. Hershberger, R. Macklin, M. Balakrishnan, N. Hill, M. McEllistrem, Phys. Rev. C 28 (1983) 2249.

[14] R.L. Macklin, R.R. Winters, N.W. Hill, J.A. Harvey, Astrophys. J. 274 (1983) 408.

[15] http://www.nndc.bnl.gov/exfor3/exfor00.htm.

[16] The n_TOF collaboration, CERN n_TOF facility: Performance Report, CERN, 2002, CERN/INTC-O-011.

[17] R. Plag, M. Heil, F. Käppeler, P. Pavlopoulos, R. Reifarth, K. Wisshak, Nucl. Instrum. Methods A 496 (2003) 425.

[18] G. Aerts, E. Berthoumiex, F. Gunsing, L. Perrot, Weighting functions for the neutron capture measurements performed at n_TOF-CERN in 2002-2003, CEA/Saclay, France, 2004. DAPNIA-04-106.

[19] W. Ratynski, F. Käppeler, Phys. Rev. C 37 (1988) 595.

[20] F.H. Fröhner, General Atomic Report, GA-8380, 1968.

[21] Z.Y. Bao, H. Beer, F. Käppeler, F. Voss, K. Wisshak, Atomic Data Nucl. Data Tables 76 (2000) 70.

[22] M.V. Bokhovko, V.N. Kononov, E.D. Poletaev, N.S. Rabotnov, V.M. Timokhov, in: S.M. Qaim (Ed.), Nuclear Data for Science and Technology, Springer, Berlin, 1992, p. 62.

[23] C.E. Rolfs, W.S Rodney, Cauldrons in the Cosmos, The University of Chicago Press, Chicago, 1996.

[24] N. Dauphas, Nature 435 (2005) 1203.

[25] M. Segawa, et al., in: A. Mengoni, et al. (Eds.), Nuclei in the Cosmos-IX (to be published in Proceedings of Science, http://pos.sissa.it). 